\title{
A 2-collinear-DoF strut with embedded negative-resistance electromagnetic shunt dampers for spacecraft micro-vibration
}

\author{
Alessandro Stabile ${ }^{1}$, Guglielmo S. Aglietti ${ }^{1}$, Guy Richardson ${ }^{2}$ \\ and Geert Smet ${ }^{3}$ \\ ${ }^{1}$ Surrey Space Centre, University of Surrey, Guildford, GU2 7XY, UK \\ ${ }^{2}$ Surrey Satellite Technology Ltd. (SSTL), Guildford, GU2 7YE, UK \\ ${ }^{3}$ ESA/ESTEC, Keplerlaan 1, PO Box 299, 2200 AG, Noordwijk, Netherlands \\ E-mail: a.stabile@surrey.ac.uk
}

\begin{abstract}
.
Micro-vibration on board a spacecraft is an important issue that affects payloads requiring high pointing accuracy. Although isolators have been extensively studied and implemented to tackle this issue, their application is far from being ideal due to the several drawbacks that they present, such as limited low-frequency attenuation for passive systems or high power consumption and reliability issues for active systems. In the present study, a novel 2-collinear-DoF strut with embedded Electromagnetic Shunt Dampers (EMSD) is modelled, analysed and the concept is physically tested. The combination of high-inductance components and negative-resistance circuits is used in the two shunt circuits to improve the EMSD micro-vibration mitigation and to achieve an overall strut damping performance that is characterised by the elimination of the resonance peaks and a remarkable FRF final decay rate of $-80 \mathrm{~dB} /$ dec. The EMSD operates without requiring any control algorithm and can be comfortably integrated on a satellite due to the low power required, the simplified electronics and the small mass. This work demonstrates, both analytically and experimentally, that the proposed
\end{abstract}


strut is capable of producing better isolation performance than other well-established damping solutions over the whole temperature range of interest.

Keywords: electromagnetic shunt damper, semi-active isolator, micro-vibration isolation Submitted to: Smart Mater. Struct. 


\section{Introduction}

Many space sensing and communication payloads such as laser communication devices and astronomical telescopes demand high pointing accuracy from the host spacecraft in order to perform their mission. Micro-vibrations are typically the main cause of limited platform stability and they are generated by several on-board subsystems and devices, such as reaction wheel assemblies, momentum wheel assemblies, control moment gyros and cryo-coolers. Developing a low-noise spacecraft bus to target a desired performance is usually one of the options to tackle this issue but it comes with a considerable increase in system cost. Moreover, reconfiguring the bus to change the transmission path is incompatible with the current trend towards lighter and cheaper structures. Contrarily, the use of isolation systems aimed at dynamically decoupling either the sensitive payload or the disturbance source from the satellite structure was proved to effectively counteract the micro-vibration issue without requiring expensive, timeconsuming design and modification of the satellite bus.

Passive isolators are mostly preferred due to their constructive simplicity, compact size and reliability. Viscoelastic materials are often used by companies in the space sector given their low cost, reduced mass and good high frequency attenuation (final slope of $-40 \mathrm{~dB} / \mathrm{dec})[1-3]$. However, the impossibility to completely eliminate the resonance peak due to their limited loss factor and the strong dependency on the operating temperature and frequency ranges make the design of viscoelastic dampers quite complex. Another well-established passive isolator is the D-Strut which includes a viscous damper specifically implemented for space applications [4,5]. Although it presents several advantages (e.g. low temperature sensitivity, high damping, linear and predictable performance) that made it favourable in many space missions, including the Hubble Space Telescope, this isolator is also characterised by high values of stiffness that 
results in a first resonance mode at high frequency. Therefore, it is still a challenge to use pure passive isolators to dampen low frequency micro-vibration onboard a spacecraft $[6]$.

On the other hand, great flexibility is offered by fully-controllable active isolators that allow to achieve more stringent conditions of isolation especially in the low frequency range $[7,8]$. Active isolators use external actuators and sensors to provide control forces and feedback signals, and for this reason they require a significant amount of power to operate $[9,10]$. In order to combine the advantages of passive and active damping, several hybrid solutions have been studied and developed. Voice coil actuators [11-14] and piezoelectric actuators [15-17] have been placed in parallel with passive systems (e.g. D-Struts, viscoelastic materials or soft springs) to produce effective microvibration isolation both in low and high frequency range. Nevertheless, hybrid systems share the same drawbacks as pure active isolators given the many accessory devices that these systems still require (e.g. sensors, actuators and power systems).

Differently from hybrid techniques, semi-active isolators might be considered mainly as passive methods with some components of the isolation system that can be actively modified. These isolators provide better mitigation performance than pure passive systems and are more reliable than pure active systems given the absence of actuators and the limited amount of power required to function. Electrorheological and magnetorheological fluids have been extensively studied in the last few decades because of the several advantages that they present such as the fast reaction time and the high loss factor [18-22]. However, very few of this type of dampers are applied in space structures due to the weight increase.

Smart materials such as piezoelectric materials [23-25] and electromagnetic transducers $[26,27]$ can be used as semi-active dampers by connecting them to shunt 
electrical circuits. In particular, the use of negative impedances in the shunt circuit aimed at cancelling or reducing the inherent electrical properties of the transducers was recently proved to considerably enhance the damping performance of these smart materials [28-30]. Furthermore, the negative-impedance circuit offers the interesting advantage of acting as a passive component (i.e. it has a constant negative magnitude) without the need of any active control and requiring only little external power to function.

This paper presents a novel concept for a 2-collinear-DoF system with embedded Electromagnetic Shunt Dampers (EMSD) for the suppression of micro-vibration disturbances in a space environment. Each EMSD includes an iron-core inductor and a negative resistance circuit that aim at reducing the overall resistance and decreasing the ratio of resistance over inductance, thus producing a damping performance that is similar to a second-order filter [31,32]. This work demonstrates both analytically and experimentally that the two-level damping obtained with two separate EMSDs and applied to a 5-kg, suspended mass is capable of eliminating the resonance peak and achieving a remarkable decay rate of $-80 \mathrm{~dB} /$ dec. A parametric trade-off is carried out to guarantee that the damping requirements are fulfilled along with the stability conditions derived from considering an operating temperature range from $-20{ }^{\circ} \mathrm{C}$ to $+50{ }^{\circ} \mathrm{C}$. The proposed strut not only outperforms other well-established isolators (e.g. viscoelastic materials and 1-DoF EMSD systems) in terms of micro-vibration mitigation, but results also extremely competitive with actively-controlled struts given the small device mass (i.e. secondary mass is less than $4 \%$ of the suspended mass), the simple electronics and the low power required to operate.

The rest of the paper is organised as follows. Section 2 presents the analytical model of the proposed strut. Section 3 describes the experimental set up. Section 4 reports 
the tests results and shows the correlation with the analytical data. The conclusions are drawn in Section 5.

\section{Analytical model}

A schematic of the system studied in this paper is shown in figure 1. This model consists of a magnet $m_{2}$ that is connected to a primary mass $m_{1}$ and to the ground via two separate springs with stiffness coefficients $k_{1}$ and $k_{2}$. The two masses can only move along their longitudinal axis, thus resulting in a 2-collinear-DoF system. By exploiting the bipolarity of a magnet, a two-level damping can be obtained using two separate electromagnets that are rigidly connected respectively to the suspended mass and to the ground. Each electromagnet is then connected to different shunt impedances thus forming two separate dampers $\left(\mathrm{EMSD}_{1}\right.$ and $\left.\mathrm{EMSD}_{2}\right)$.

It is noted that real micro-vibration sources produce disturbances in all six degrees of freedom whereas the proposed isolator counteracts a disturbance only along a single degree of freedom (since the strut's two degrees of freedom are collinear). However, the evaluation of the damper performance of a single strut allows it to be compared with other damping solutions $[1,31]$.

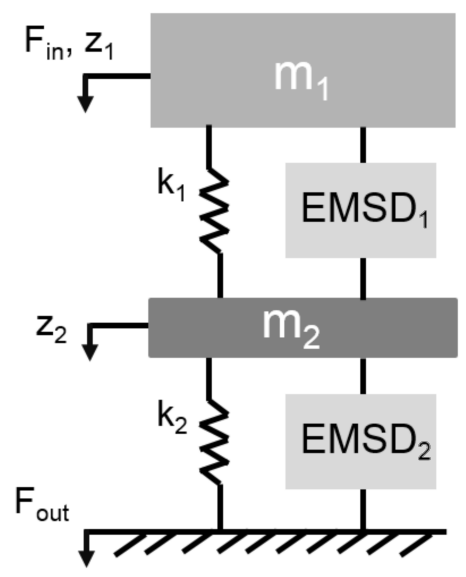

Figure 1: Schematic representation of the 2-collinear-DoF model 


\subsection{State-space modelling}

EMSDs provide a damping force due to the combination of two different physics phenomena that are described by the Faraday-Lenz law and the Lorentz force law (see [31] for more details). Through the assumptions of constant magnetic field seen by the conductive material (due to the relative displacement being in the order of magnitude of tenths of a millimetre for a micro-vibration load case), linear trend of the magnetic field along the radial axis inside the small cross section of the electromagnet, and relative motion between the conductor and the magnet to be only along the z-axis, the FaradayLenz law and the Lorentz force law can be respectively simplified as:

$$
\begin{aligned}
& V_{0}=2 \pi n_{t} r_{a v g} \bar{B}_{r} v_{z}=K_{d} v_{z} \\
& \vec{F}_{d}=-2 \pi n_{t} r_{a v g} \bar{B}_{r} I \hat{z}=-K_{d} I \hat{z}
\end{aligned}
$$

where $\hat{z}$ is the vertical axis, $v_{z}$ is the relative velocity between the magnet and the coil along the $\hat{z}$ axis, $n_{t}$ is the number of turns of the coil, $r_{\text {avg }}$ is the average radius of the conductor, $\bar{B}_{r}$ is the average radial component of the magnetic field through the coil cross section. $K_{d}$ is defined as the electro-mechanical transducer coefficient.

These two laws considered together demonstrate that the force produced by a permanent magnet which moves close to a conductive material is proportional in magnitude and opposite in direction to their relative velocity. Therefore, this kind of force opposes the movement of the magnet and acts like a damping force.

The system showed in figure 1 can be modelled via a fully-coupled system where (1) and (2) are integrated with the equation of motion and the Kirchhoff's voltage law associated with the electric circuit. Through the assumptions of micro-vibration and steady-state conditions, this system can be linearised via a state-space representation. The state vector consists of six state variables: displacements $\left(z_{1}, z_{2}\right)$, velocities $\left(\dot{z}_{1}, \dot{z}_{2}\right)$, 
and circuit currents $\left(I_{1}, I_{2}\right)$. The state space model can be written as:

$$
\left[\begin{array}{c}
\dot{z}_{1} \\
\dot{z}_{2} \\
\ddot{z}_{1} \\
\ddot{z}_{2} \\
\dot{I}_{1} \\
\dot{I}_{2}
\end{array}\right]=\left[\begin{array}{ccc}
0_{2,2} & \mathcal{I}_{2} & 0_{2,2} \\
\mathcal{K} & 0_{2,2} & \mathcal{C}_{m} \\
0_{2,2} & \mathcal{C}_{L} & \mathcal{R}
\end{array}\right]\left[\begin{array}{c}
z_{1} \\
z_{2} \\
\dot{z}_{1} \\
\dot{z}_{2} \\
I_{1} \\
I_{2}
\end{array}\right]+\left[\begin{array}{c}
0 \\
0 \\
1 / m_{1} \\
0 \\
0 \\
0
\end{array}\right] F_{\text {in }}
$$

where $O_{2,2}$ and $\mathcal{I}_{2}$ are respectively the $2 \times 2$ null and identity matrices. $\mathcal{K}$ and $\mathcal{C}_{m}$ represent the stiffness and damping matrices in the equation of motion and are defined as:

$$
\begin{aligned}
& \mathcal{K}=\left[\begin{array}{cc}
-k_{1} / m_{1} & k_{1} / m_{1} \\
k_{1} / m_{2} & -\left(k_{1}+k_{2}\right) / m_{2}
\end{array}\right] \\
& \mathcal{C}_{m}=\left[\begin{array}{cc}
-K_{d} / m_{1} & 0 \\
K_{d} / m_{2} & -K_{d} / m_{2}
\end{array}\right]
\end{aligned}
$$

whereas $\mathcal{C}_{L}$ and $\mathcal{R}$ are representative of the induced voltages and the resistances that are associated with the two electric circuits and are defined as:

$$
\begin{aligned}
& \mathcal{C}_{L}=\left[\begin{array}{cc}
K_{d} / L_{1} & -K_{d} / L_{1} \\
0 & K_{d} / L_{2}
\end{array}\right] \\
& \mathcal{R}=\left[\begin{array}{cc}
-R_{1} / L_{1} & 0 \\
0 & -R_{2} / L_{2}
\end{array}\right] .
\end{aligned}
$$

In particular, $R_{1}$ and $L_{1}$ are the circuit features related to the $\mathrm{EMSD}_{1}$, while $R_{2}$ and $L_{2}$ are related to the $\mathrm{EMSD}_{2}$.

In order to assess the damping performance, it is necessary to evaluate the Transfer Function (TF) between the input force $F_{i n}$ and the force transmitted to the satellite 
structure. The latter can be obtained by setting the output vector as:

$$
Y=\left[\begin{array}{llllll}
0 & k 2 & 0 & 0 & 0 & K_{d}
\end{array}\right]\left[\begin{array}{c}
z_{1} \\
z_{2} \\
\dot{z}_{1} \\
\dot{z}_{2} \\
I_{1} \\
I_{2}
\end{array}\right]+\left[\begin{array}{l}
0
\end{array}\right] F_{\text {in }}
$$

and consequently the force TF can be evaluated after converting this single-input-singleoutput system to the frequency domain.

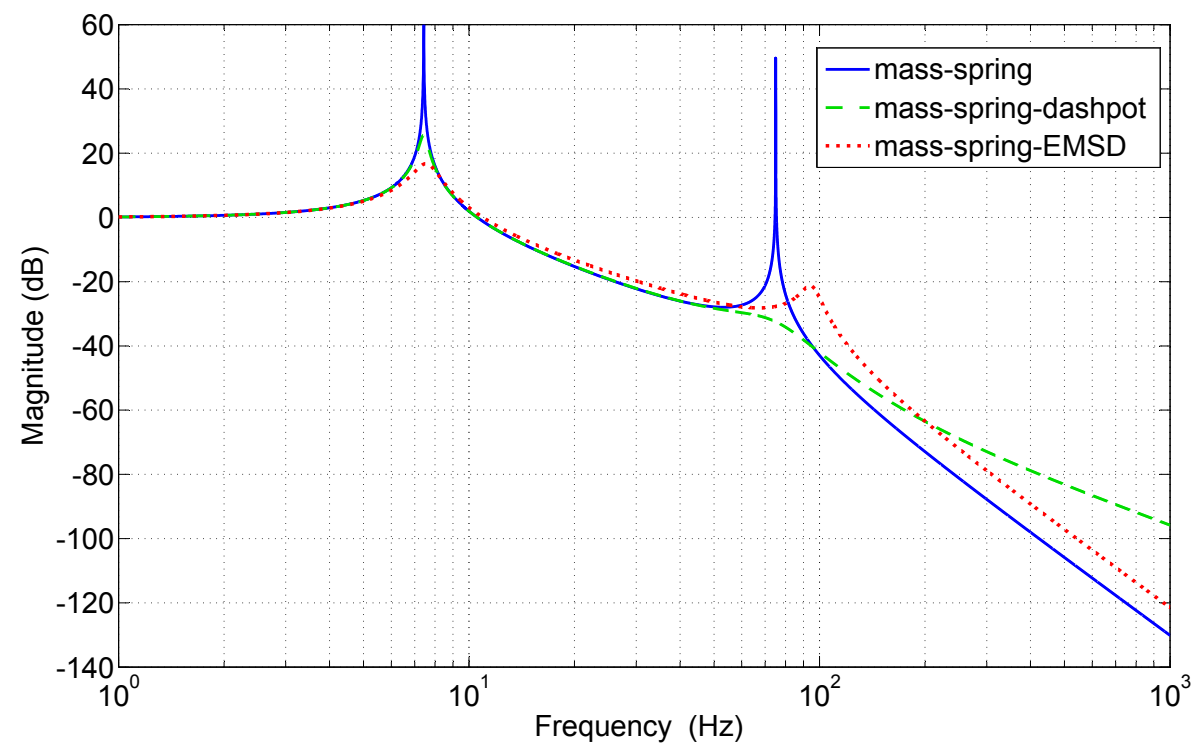

Figure 2: Comparison between TFs associated with three different 2-DoF systems: mass-spring system characterised by two couples of complex-conjugate poles (solid line), mass-spring-dashpot system characterised by two additional real zeros (dashed line) and mass-spring-EMSD system that introduces two extra real poles thus restating the final decay rate to $-80 \mathrm{~dB} / \mathrm{dec}$ (dotted line).

In order to better understand how the introduction of two EMSDs affects the system response, an example with the TFs associated with three different system configurations is shown in figure 2. A typical TF for a two-masses-two-springs system is characterised 
by two resonance peaks (i.e. two couples of complex-conjugate poles) and a final roll-off slope of $-80 \mathrm{~dB} / \mathrm{dec}$ (solid line). Adding viscous dampers in parallel to the springs (dashed line) would help reducing the force amplification at the resonance peaks, but it would also reduce the high-frequency attenuation due to the introduction of two real zeros in the $\mathrm{TF}$ that brings the slope to $-40 \mathrm{~dB} / \mathrm{dec}$ (i.e. each real zero increases the decay rate by $+20 \mathrm{~dB} / \mathrm{dec})$. The two-level damping made of two separate EMSDs that is proposed in this paper is capable of combining the advantages of viscous dampers (i.e. considerable reduction of the resonance peaks by decreasing the overall circuit resistance) and of 2-DoF mass-spring systems, since the relatively low resistance-overinductance ratio introduces a real pole per EMSD that restores the final roll-off slope to $-80 \mathrm{~dB} / \mathrm{dec}$ (dotted line).

\subsection{Strut configuration}

The main feature of the isolator presented in this paper is the improved damping performance without the need to increase the secondary mass. This goal has been achieved by analysing two key aspects of this system such as the magnetic assembly and the shunt circuit.

The magnetic assembly strongly affects the damping force $F_{d}$ through the electromechanical transducer coefficient $K_{d}$ which depends on the radial component of the magnetic field, as shown in (1). A configuration trade-off of the magnetic assembly was previously conducted through the software COMSOL Multiphysics [31] with the goal of increasing the ratio of $K_{d}$ over magnet mass. The chosen configuration can be seen in figure 3. The magnet stack is characterised by two central regions where the radial component of the magnetic field is considerably enhanced due to the magnets facing each other with opposite polarity. This configuration presents also two extra poles at the stack's extremities that can be exploited by two other coils. In this study each 
electromagnet is made of two coils connected together, as it can be observed from figure 3a.

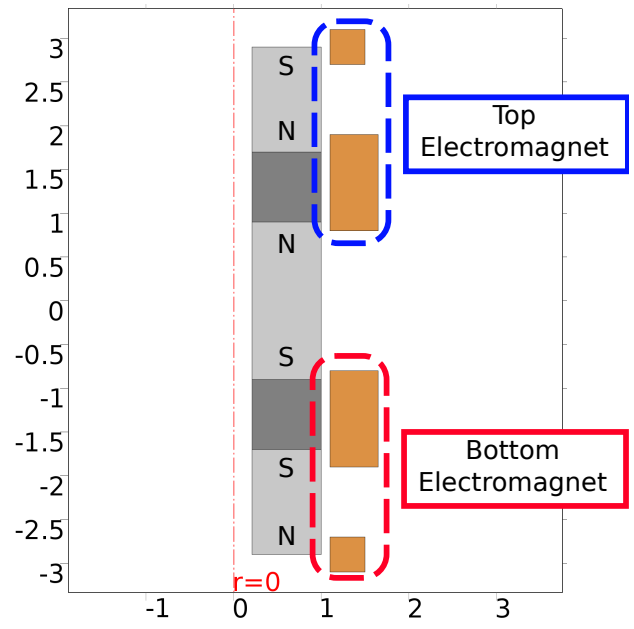

(a)

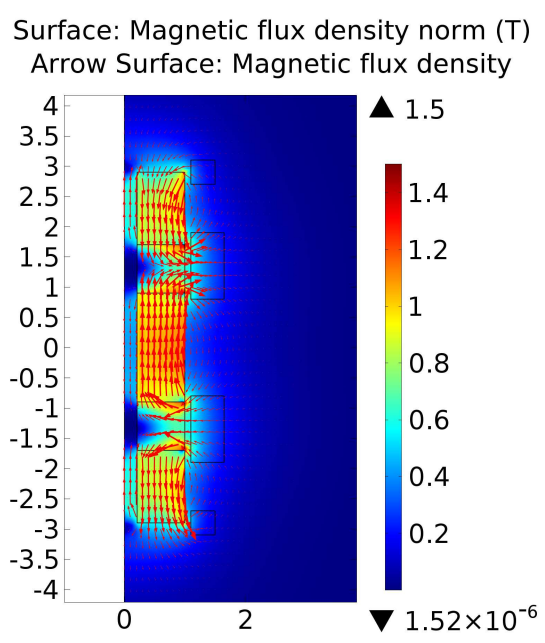

(b)

Figure 3: Magnetic assembly analysis carried out through COMSOL Multiphysics using a 2D-axisymmetric formulation. (a) disposition of the magnets and yokes with relative magnetic polarization; (b) magnetic flux density.

The micro-vibration isolation of the proposed strut can also be improved by modifying the electrical features of the shunt circuit. As already stated above, reducing the overall resistance has a beneficial effect on the amplification at the resonance peaks, whereas reducing the ratio $R / L$ produces the shift of the extra pole toward lower frequencies which results in a better high frequency isolation performance. Both tasks can be achieved by combining the use of an inductor and a negative resistance in the shunt circuit. In particular, the inductor should be characterised by having a high ratio of inductance over internal resistance, and for this reason iron-core inductors are usually preferable (they can present values of inductance in the order of $\mathrm{mH}$ and inherent resistances in the order of tenths of a Ohm). It is important to notice that increasing the inductance produces the same effect that can be achieved by increasing the secondary mass (having assumed that all the other parameters, e.g. $\bar{B}_{r}$, remain constant). Figure 4 shows for example that changing the inductances $L_{1}$ and $L_{2}$ from $1 \mathrm{mH}$ to $5 \mathrm{mH}$ (red 
curve) would generate a high-frequency attenuation that is comparable with the system where the secondary mass is set to $3.5 \mathrm{~kg}$ (purple curve). Therefore, having a large inductance can be beneficial not only in terms of performance but also at system level since the mass of the isolator can be kept at a minimum.

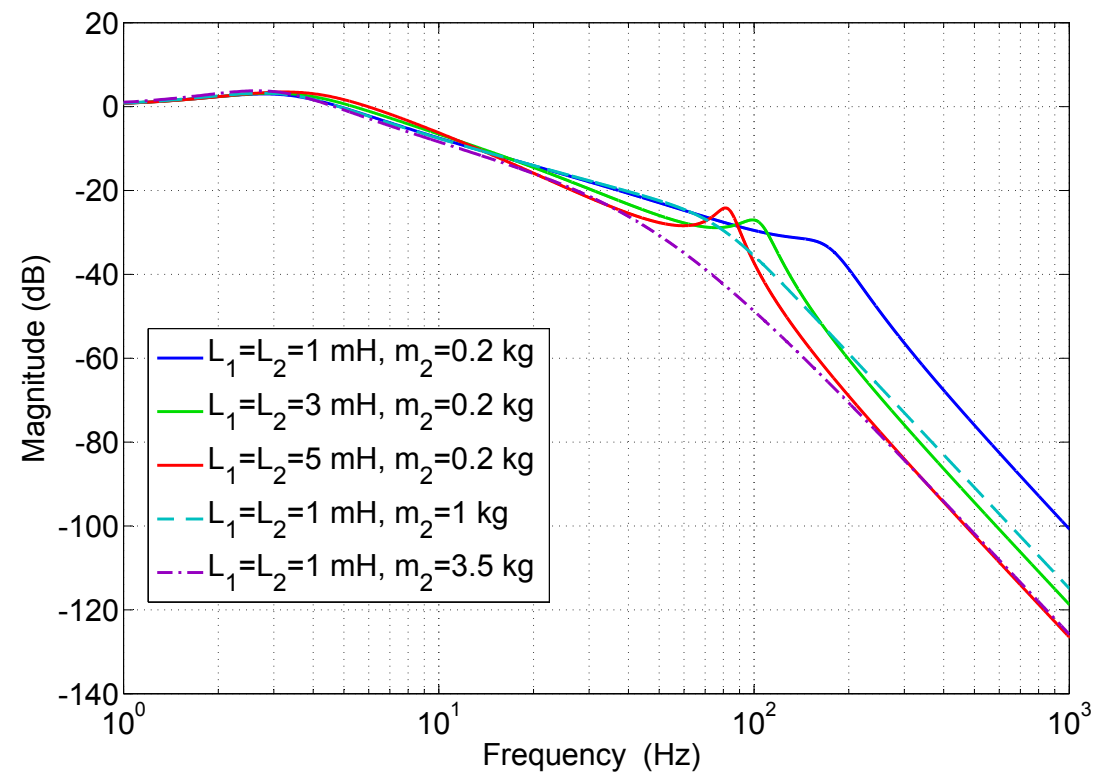

Figure 4: Effect of changing the inductance and the secondary mass on the system damping performance

On the other hand, the negative resistance is created by utilising an analogue circuit called negative impedance converter [33]. It consists of a single operational amplifier (op-amp) and three resistors that are connected as shown in figure 5. The equivalent resistance of the shunt is:

$$
R_{s}=-R_{x}\left(\frac{R_{z}}{R_{y}}\right) .
$$

By considering the resistances $R_{y}$ and $R_{z}$ to be equal to each other, the shunt produces a negative resistance $R_{s}=-R_{x}$. Although reducing the overall resistance has the effect of enhancing the damping performance by increasing the electric current, the use of the negative impedance converter is limited by the fact that this system would 
result unstable if the negative resistance becomes greater in magnitude than the positive one. This condition can occur due to the typical temperature fluctuations of a space mission (this aspect will be taken into account in the next paragraph). For this reason the use of an inductor in series with the electromagnet becomes crucial to further reduce the ratio $R / L$ and so increase the damper performance.

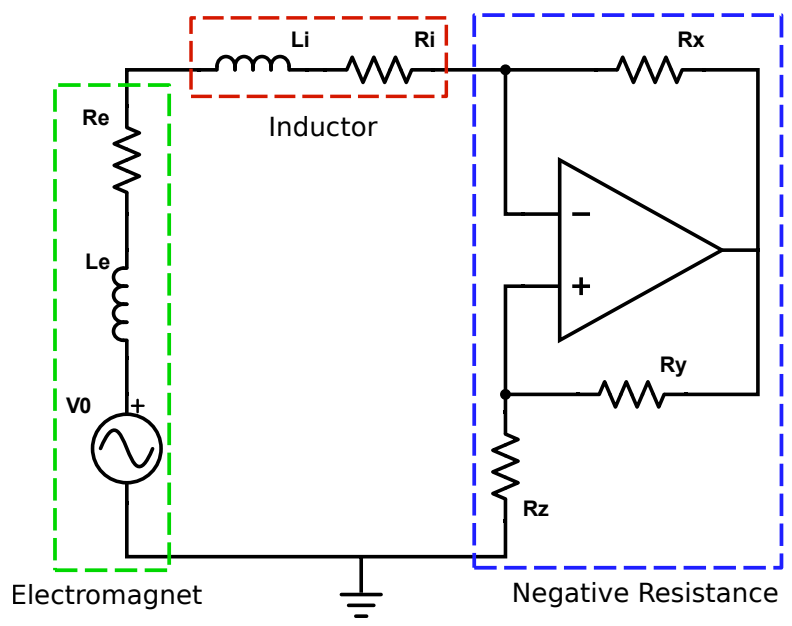

Figure 5: Schematic of the EMSD electric circuit

\subsection{Parametric trade-off}

Isolation systems are usually affected by the environmental conditions at which a satellite operates (e.g. viscoelastic materials are strongly dependent on the surrounding temperature). An EMSD (made uniquely of metal parts such as Nd-Fe-B magnets and copper) has the advantage that only the copper resistivity of the coils and the residual induction of the Nd-Fe-B magnet are notably affected by the temperature change and are respectively characterised by the thermal coefficients $0.00386{ }^{\circ} \mathrm{C}^{-1}$ and -0.0012 ${ }^{\circ} \mathrm{C}^{-1}$.

The proposed isolator has been designed to provide good micro-vibration mitigation within the operating temperature range without requiring any active control. The suspended mass $m_{1}$ has been chosen to be $5 \mathrm{~kg}$ and corresponds approximately to the 
mass of typical reaction wheel assemblies (e.g. two 100SP-O reaction wheels used by Surrey Satellite Technology Ltd.). Reaction wheels work usually within a temperature range that spans from $-20{ }^{\circ} \mathrm{C}$ to $+50{ }^{\circ} \mathrm{C}[2,3]$. A parametric trade-off has been conducted in order to meet the following goals throughout the whole temperature range of interest:

- Maximum amplification below $6 \mathrm{~dB}$

- Corner frequency at $10 \mathrm{~Hz}$ or below

- At least $-40 \mathrm{~dB}$ at $100 \mathrm{~Hz}$.

Along with these goals, it is crucial to guarantee the stability of the system for all the operating conditions. An EMSD would become unstable if the negative resistance is greater in magnitude than the positive one $\left(R_{e}+R_{i}\right)$ and this could happen at the lowest temperature range limit [31]. In order to prevent the system from becoming unstable, the total resistance at the temperature of $-20{ }^{\circ} \mathrm{C}$ was set to a minimum value $0.2 \Omega$.

The trade-off was carried out by analysing the system at three different temperatures: $-20{ }^{\circ} \mathrm{C},+50{ }^{\circ} \mathrm{C}$ and the range mid point, $+15^{\circ} \mathrm{C}$. The analysis included few assumptions that simplified the integration between the mechanical, electromagnetic and thermal domains. First of all, similarly to the work presented in [31] it was assumed that all the components of the electric circuit have the same temperature of the surrounding environment. In this way it was possible to study the system in steady state conditions. Secondly, it was assumed that the two springs were identical $\left(k_{1}=k_{2}\right)$, as well as the electrical properties of the two electromagnetic circuits $\left(R_{1}=R_{2}\right.$ and $L_{1}=L_{2}$ ). The final choice of the parameter set is reported in table 1 .

A comparison of the force TFs between the system without damping (i.e. the shunt circuits were disconnected and the electromagnets were in open-circuit conditions) and the system with the two EMSDs is shown in figure 6. It can be observed that the 


\begin{tabular}{|c|c|c|c|c|}
\hline Property & & \multicolumn{3}{|c|}{ Value } \\
\hline Suspended mass, $\mathrm{m}_{1}(\mathrm{~kg})$ & & \multicolumn{3}{|c|}{5} \\
\hline Magnet mass, $\mathrm{m}_{2}(\mathrm{~kg})$ & & \multicolumn{3}{|c|}{0.18} \\
\hline Spring Stiffness, $k_{1}=k_{2}(\mathrm{~N} / \mathrm{m})$ & & \multicolumn{3}{|c|}{2000} \\
\hline Coil Inductance, $L_{1}=L_{2}(\mathrm{mH})$ & & \multicolumn{3}{|c|}{8} \\
\hline Shunt Resistance, $\mathrm{R}_{s}(\Omega)$ & & \multicolumn{3}{|c|}{-3.0} \\
\hline & Temp. & $-20^{\circ} \mathrm{C}$ & $15^{\circ} \mathrm{C}$ & $50^{\circ} \mathrm{C}$ \\
\hline Inductor Resistance, $\mathrm{R}_{i}(\Omega)$ & & 0.35 & 0.40 & 0.45 \\
\hline Coil Resistance, $\mathrm{R}_{e}(\Omega)$ & & 2.85 & 3.25 & 3.65 \\
\hline E-M Transducer Coefficient, $\mathrm{K}_{d}(\mathrm{~N} / \mathrm{A})$ & & 11.28 & 10.79 & 10.29 \\
\hline
\end{tabular}

Table 1: Final choice of the parameter set obtained through a trade-off

use of the EMSDs produces the desired reduction of the two resonance peaks and the final roll-off slope of $-80 \mathrm{~dB} / \mathrm{dec}$. The maximum amplification is kept below $6 \mathrm{~dB}$ in all three cases studied, and the corner frequency, although moving slightly within the temperature range, does not exceed $10 \mathrm{~Hz}$. Also, the force attenuation at $100 \mathrm{~Hz}$ is greater than $40 \mathrm{~dB}$. Therefore, the three aforementioned goals are all met in the tradeoff analysis. It is noted that the shift of the first resonance peak within the temperature range of interest is in agreement to what already reported in [31].

\section{Experimental set-up}

A laboratory test rig was built in house and mounted on a Kistler Table (KT), as it can be seen in figure 7a, and it represents a physical proof of concept of the proposed strut. The experimental set-up was designed to meet the requirement of the two masses having only a vertical displacement, while trying to minimise displacements/rotations along the other five DoFs. In order to do that, steel flexures were connected between 


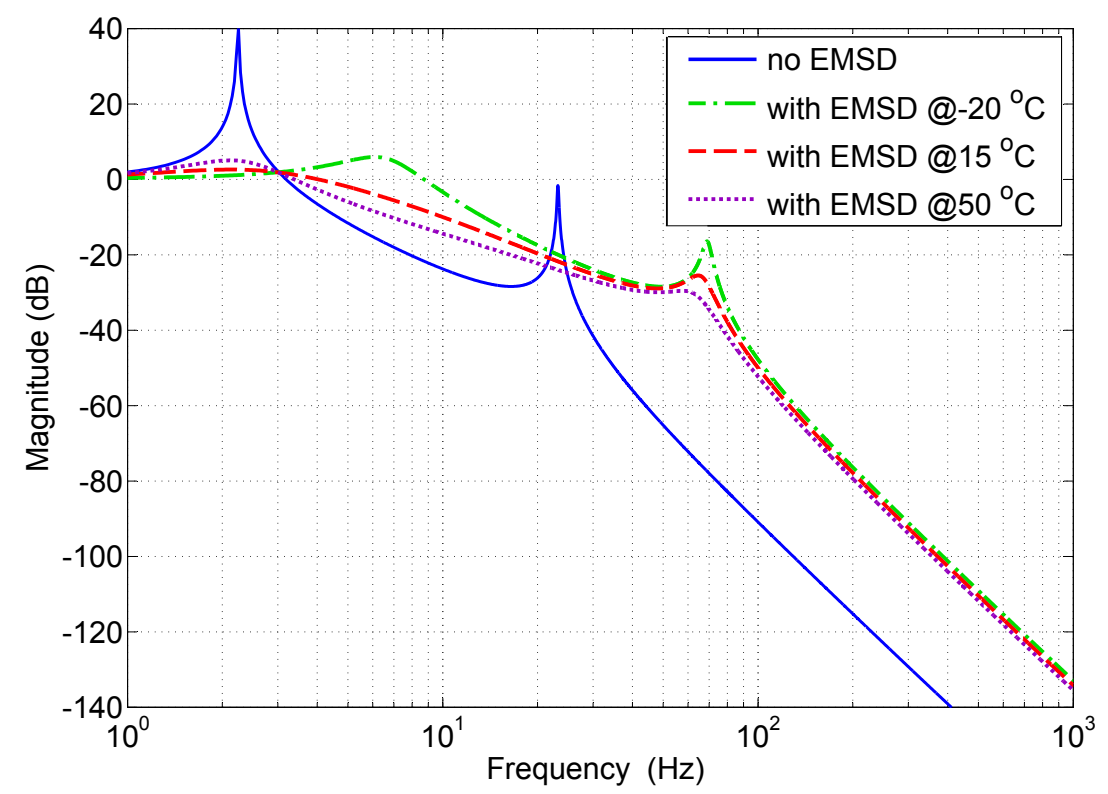

Figure 6: Comparison of the analytical solution of the force TFs between the system without EMSD (i.e. electromagnets in open-circuit conditions) and the system with EMSD at three different temperatures.

both masses and the test rig's support structure. Although their use is necessary to guarantee the 2-collinear-DoF system, the inclusion of the flexures produces a slight change in the analytical model of the test rig. In this experimental configuration the primary and secondary masses are further connected to the ground through the flexures that can be represented with vertical springs called respectively $k_{b f}$ and $k_{s f}$ (see figure 7b). The system model reported in (3) can be adapted to the actual configuration by solely changing the matrix $\mathcal{K}$ as:

$$
\mathcal{K}=\left[\begin{array}{cc}
-\left(k_{1}+2 \cdot k_{b f}\right) / m_{1} & k_{1} / m_{1} \\
k_{1} / m_{2} & -\left(k_{1}+k_{2}+2 \cdot k_{f s}\right) / m_{2}
\end{array}\right] .
$$

A model of the test rig was evaluated through a FEM analysis on Nastran. This analysis showed that the extra load paths introduced with the flexures and transmitted to the KT considerably modify the force TF altering the expected final roll-off slope of $-80 \mathrm{~dB} / \mathrm{dec}$, as it can be observed on figure 8 . Moreover, disturbances coming from the 
flexures' secondary modes are recorded by the KT from about $200 \mathrm{~Hz}$, thus affecting the force TF.

In order to tackle both issues, a Multiaxial Force Sensor (MFS) was inserted on top of the KT and underneath the support that holds the bottom electromagnet and the spring $k_{2}$ (see detail in figure 7a). The force TF between the input force and the force recorded on the MFS has been evaluated with Nastran (see figure 8). It is characterised by the elimination of the high-frequency modes associated with the flexures and by almost entirely overlapping with the $\mathrm{TF}$ of the theoretical 2-collinear-DoF strut described in (3). Therefore, with the inclusion of the MFS in the test rig it is possible to obtain an almost accurate assessment of the isolation performance of the proposed strut. The main differences between the two curves can be observed at low frequency where the TF associated with the MFS starts below $0 \mathrm{~dB}$ because part of the low-frequency force is conveyed to the ground through the rig's walls. Also, the first resonance frequency slightly shifts toward higher frequencies due to the extra stiffness $k_{b f}$ and $k_{s f}$ produced by the flexures. It is noted that a small amount of viscous damping (less than $6 \%$ of the critical damping) has been added to both Nastran and Matlab models in order to reflect the inherent mechanical damping that was observed in previous studies on a similar version of this test rig [31]. This damping naturally occurs in a mechanical assembly and can be produced by several factors such as micro-friction between adjacent components, air resistance and hysteresis damping in the steel flexures.

Another fundamental aspect of the proposed strut is the low system mass compared to the suspended mass. The magnetic assembly weighs $180 \mathrm{~g}$ which represents less than $4 \%$ of the total suspended mass. The dummy mass of $5 \mathrm{~kg}$ is obtained through the combination of steel plates bolted together and aluminium/plastic harnesses. In order to simulate microgravity, the primary mass was offloaded using elastic cords characterised 
by a sub-hertz resonance frequency.

Two different tests were performed on this test rig. First, a sine sweep was applied to the dummy mass through a suspended mini-shaker that was connected to its centre. The scope of this test was to verify the system behaviour at a wide frequency range. In fact, the mini-shaker is able to introduce a high input force throughout the frequency range of interest that allows the output signal to mostly overcome the background noise and reach a maximum attenuation level of about $-80 \mathrm{~dB}$. The second test involved the use of a real reaction wheel provided by Surrey Satellite Technology Ltd. A spinning reaction wheel produces a broadband disturbance due to several microscopic defects or imperfections that are present inside the device (e.g. flywheel unbalance or cage disturbance). This test aimed at demonstrating the capability of the EMSD to respond to a broadband input signal while maintaining its linear behaviour.

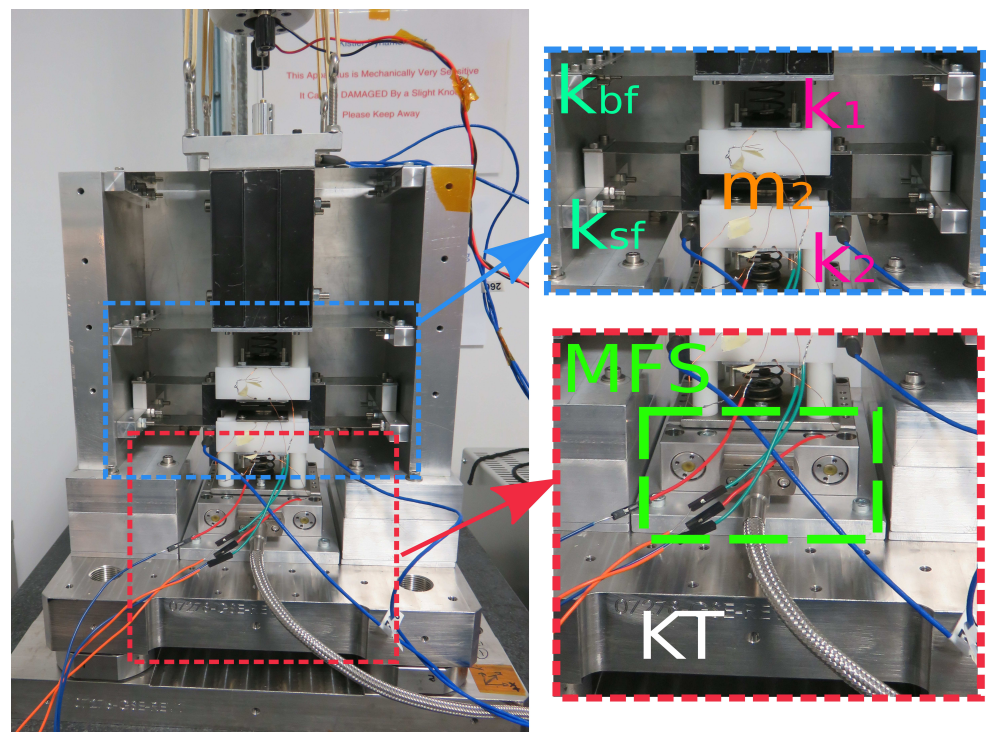

(a)

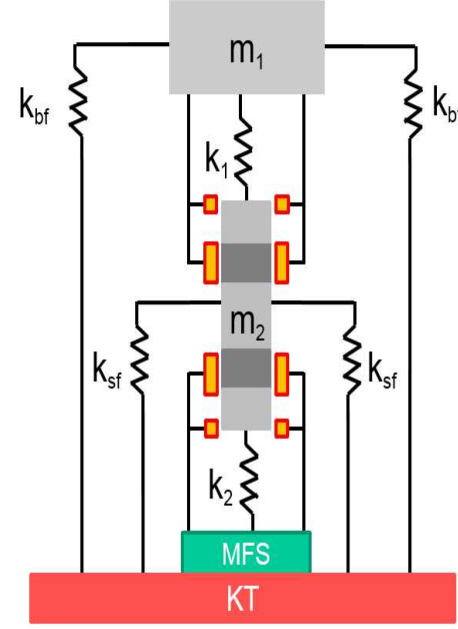

(b)

Figure 7: Experimental test rig. In particular, $K_{b f}$ and $K_{s f}$ are the equivalent stiffnesses of the flexures connected respectively to the top mass $m_{1}$ and the magnet mass $m_{2}, k_{1}$ and $k_{2}$ represent the two springs that connect $m_{2}$ respectively to $m_{1}$ and to the ground, MFS is the multiaxial sensor placed on top of the KT. (a) test rig mounted on the KT; (b) Schematic representation of the test rig 


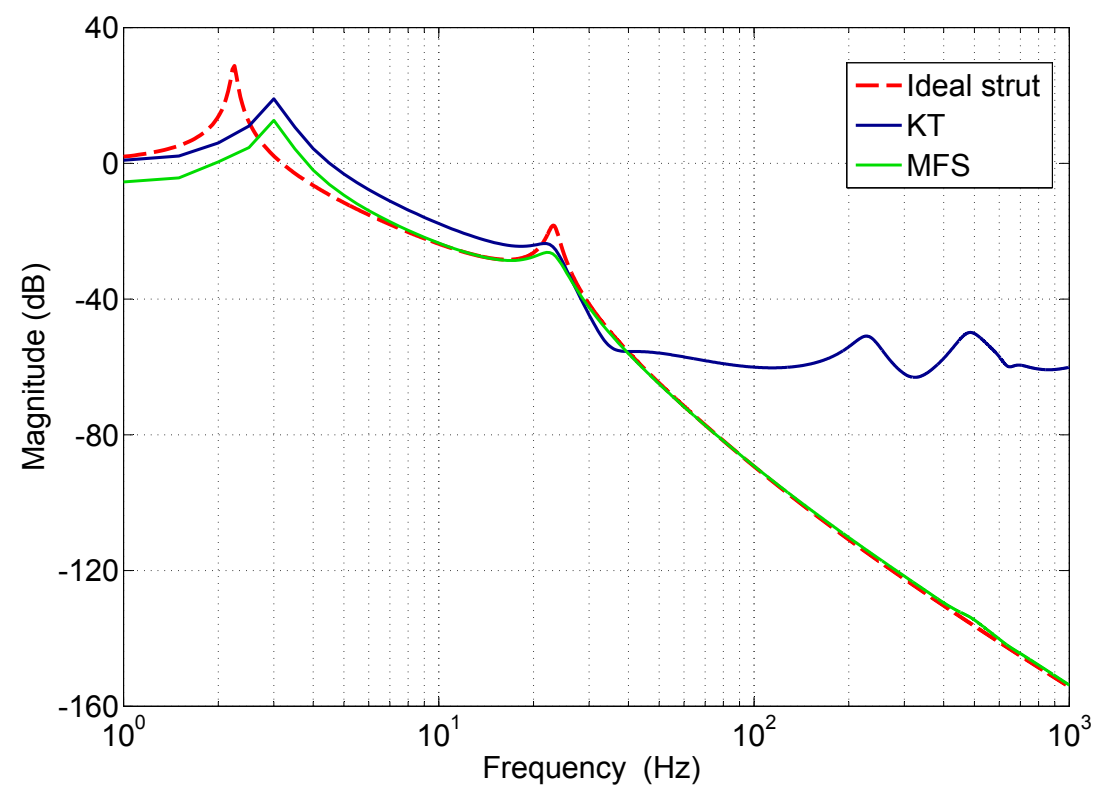

Figure 8: Comparison of the TFs between the analytical solution of the ideal strut (evaluated on Matlab) and the FEM results obtained through Nastran and relative to the KT and the MFS

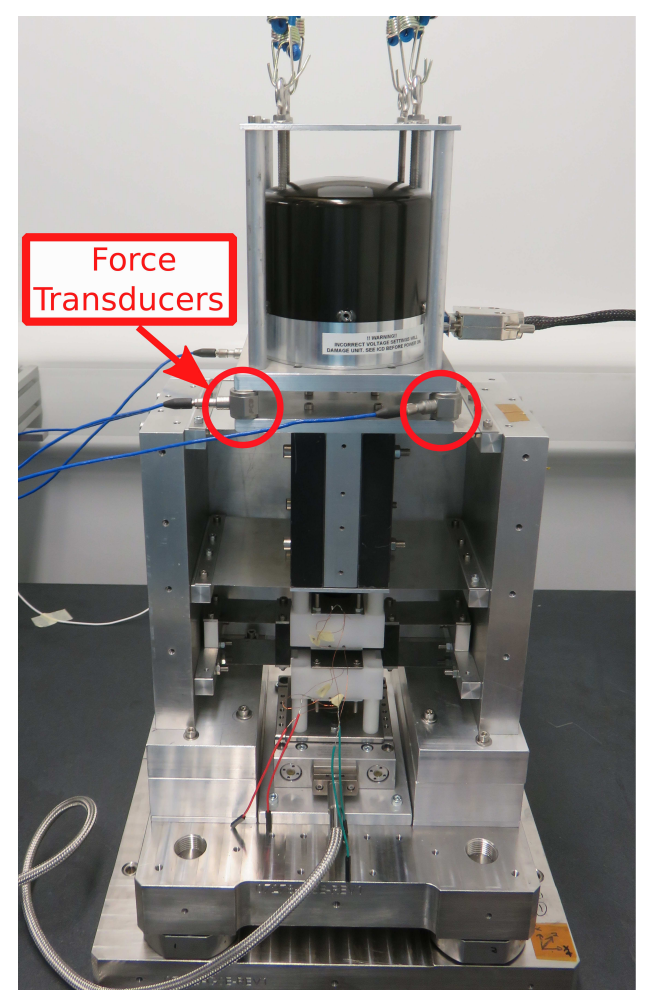

Figure 9: Test set-up with 10SP reaction wheel mounted on top of the dummy mass 


\section{Experimental results and discussions}

\subsection{Sine sweep with mini-shaker}

The KT is placed on top of an isolation system (a suspension system that acts as a low pass filter) characterised by an $8-\mathrm{Hz}$ resonance mode that required the tests to be divided into two different sine sweeps: a low-frequency one with the KT locked to the ground, and a high-frequency one with the isolation system in operation. The minishaker was controlled through a force feedback loop to generate first a 0.1-N-amplitude sinusoidal input force sweeping from $1 \mathrm{~Hz}$ to $20 \mathrm{~Hz}$, and then a sinusoidal input force with amplitude linearly increasing from $1 \mathrm{~N}$ at $10 \mathrm{~Hz}$ to $5 \mathrm{~N}$ at $400 \mathrm{~Hz}$. The input force of the first sine sweep was set to be $0.1 \mathrm{~N}$ so as to prevent the flexures from operating in the nonlinear region due to relatively large displacements (e.g. in the order of millimetres). The lab temperature was approximately $20{ }^{\circ} \mathrm{C}$ and the parametric set in the analytical model was tuned to account for this temperature. The comparison between the experimental results and the analytical data is shown in figure 10 . The background noise represents the environmental disturbances measured by MFS and it was recorded at the beginning of the test campaign. From figure 10a the comparison between the force TFs associated with the KT and MFS for a system without EMSD can be observed. In particular, the first two resonance modes are clearly visible, and above $30 \mathrm{~Hz}$ it is noted that the force TF associated with the MFS shows the expected final slope of $-80 \mathrm{~dB} / \mathrm{dec}$ up until it reaches the background noise level. Good correlation can also be seen for the system with EMSD (see figure 10b). As already shown in figure 6, the $\mathrm{TF}$ is characterised by the elimination of the first resonance peak, cut-off frequency below $10 \mathrm{~Hz}$ and (for the MFS case) roll-off slope of -80 dB/dec and attenuation greater than $40 \mathrm{~dB}$ at $100 \mathrm{~Hz}$.

The analytical model was further evaluated through the analysis of two other TFs. 
The relations between the input force and the mass velocity (for the case without the EMSD) and the induced current (for the system with the EMSD) can be obtained by modifying the output vector in (8) as follows:

$$
Y=\left[\begin{array}{c}
Y_{v 1} \\
Y_{v 2} \\
Y_{I 1} \\
Y_{I 2}
\end{array}\right]=\left[\begin{array}{cccccc}
0 & 0 & 1 & 0 & 0 & 0 \\
0 & 0 & 0 & 1 & 0 & 0 \\
0 & 0 & 0 & 0 & 1 & 0 \\
0 & 0 & 0 & 0 & 0 & 1
\end{array}\right]\left[\begin{array}{c}
z_{1} \\
z_{2} \\
\dot{z}_{1} \\
\dot{z}_{2} \\
I_{1} \\
I_{2}
\end{array}\right]+\left[\begin{array}{c}
0 \\
0
\end{array}\right] F_{i n}
$$

where $Y_{v 1}$ and $Y_{v 2}$ represent respectively the velocity of the primary mass and the magnetic assembly while $Y_{I 1}$ and $Y_{I 2}$ are the induced currents in the top and bottom electromagnets. The velocities are obtained after integrating the acceleration signals measured through accelerometers placed on top of the dummy mass and on the sides of the magnetic assembly. The currents are evaluated using an oscilloscope probe connected to the ends of each electromagnet. The comparison of these analytically-obtained TFs with the experimental data is shown respectively in figures 11 and 12 . The use of different kind of sensors that has allowed to analyse different aspects of the damping system and the good correlation that can be observed among all the presented TFs are further confirmations of the accuracy of the analytical model and also the correct functioning of the negative-resistance circuits.

\subsection{Broadband signal with reaction wheel}

The reaction wheel used for this test belongs to the 10SP series made by Surrey Satellite Technology Ltd (this reaction wheel was chosen in place of the one from the 100SP series due to availability issues at the time of the tests). The 10SP reaction wheel has a mass of approximately $1 \mathrm{~kg}$ and in order to compensate for the increased mass one of the 


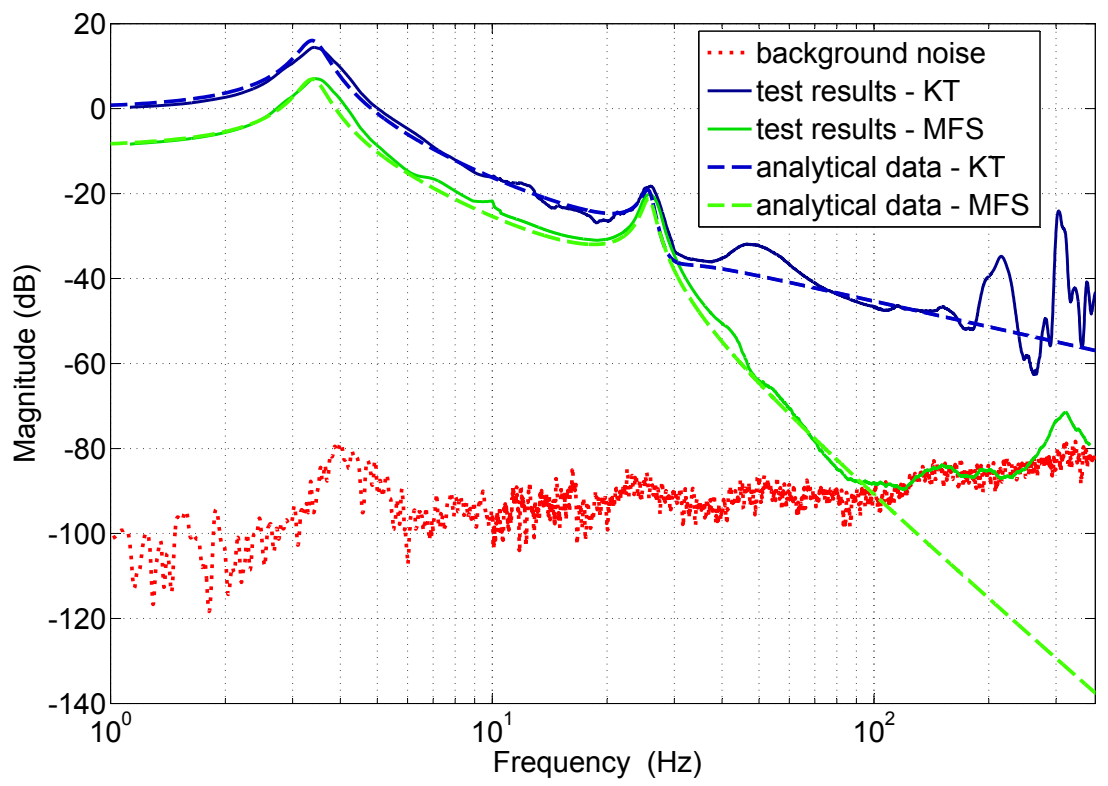

(a)

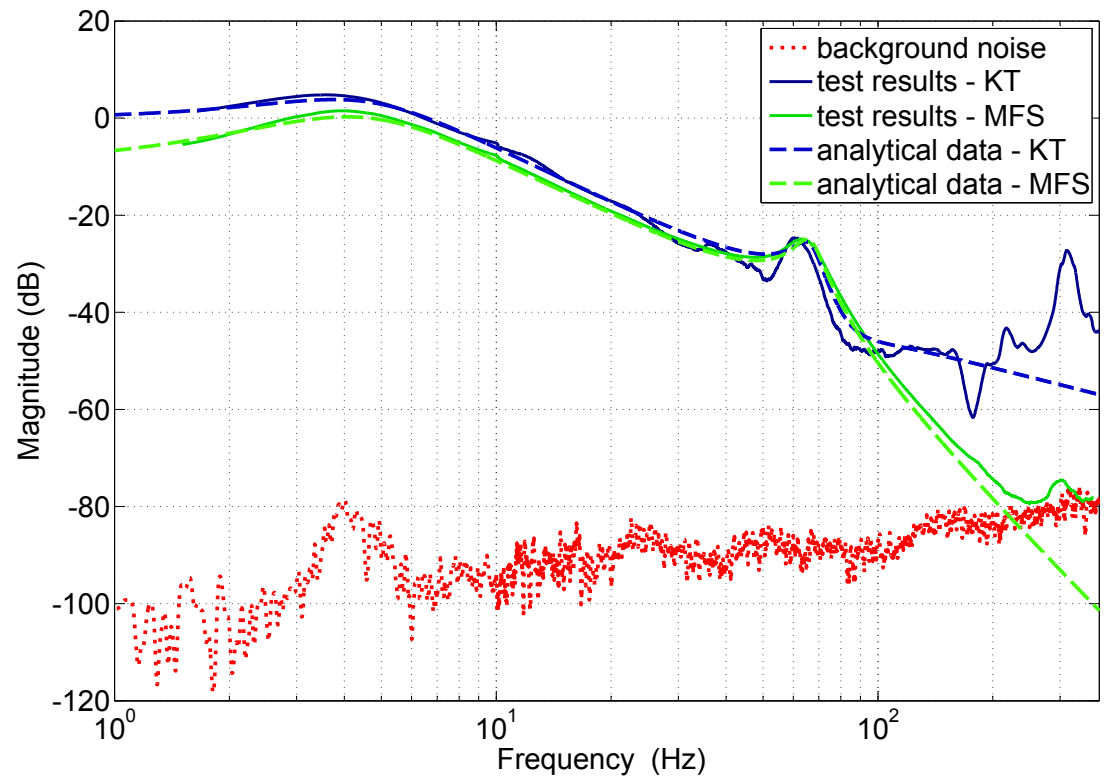

(b)

Figure 10: Comparison between the test results and the analytical model. The solid lines represent the test results, whereas the dashed lines are relative to the analytical model. The background noise is also included. (a) system without EMSD; (b) system with EMSD. 
(a)

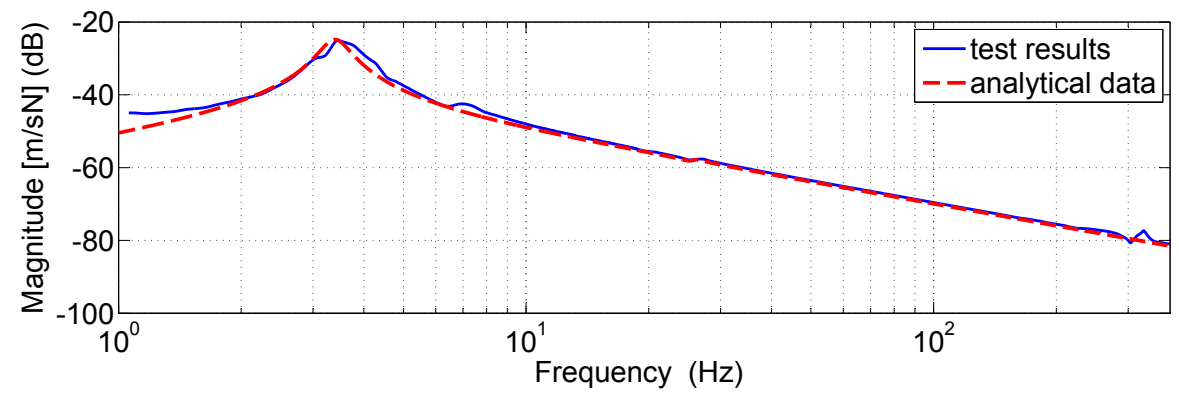

(b)

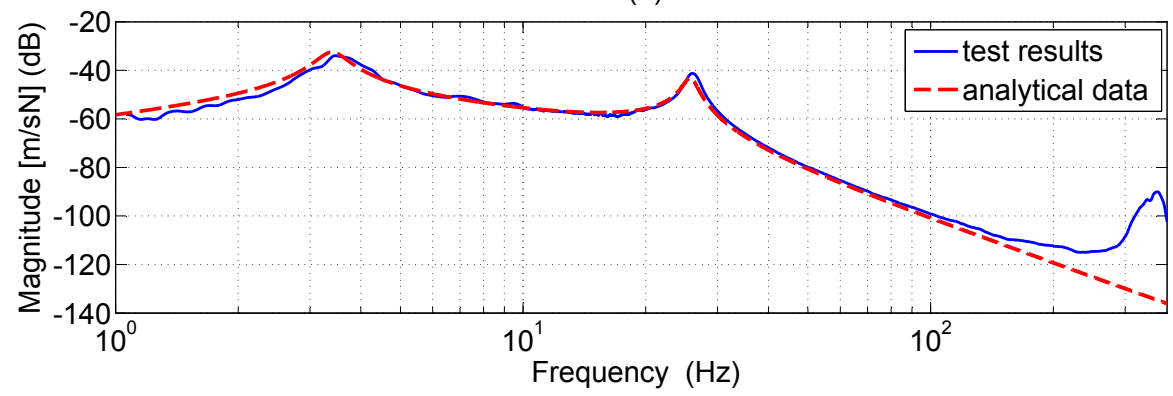

Figure 11: TF between the input force and the mass velocity. The system considered is without EMSDs. (a) primary mass $m_{1}$; (b) magnet mass $m_{2}$

(a)

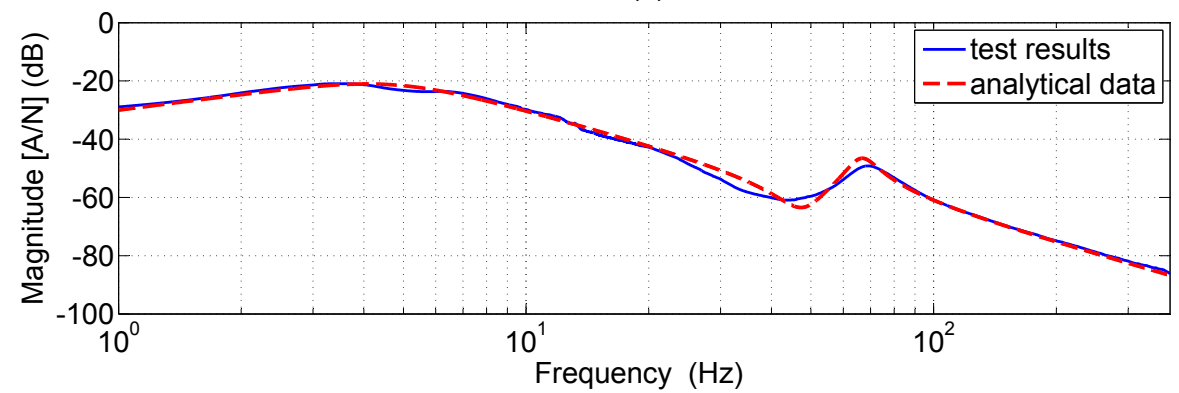

(b)

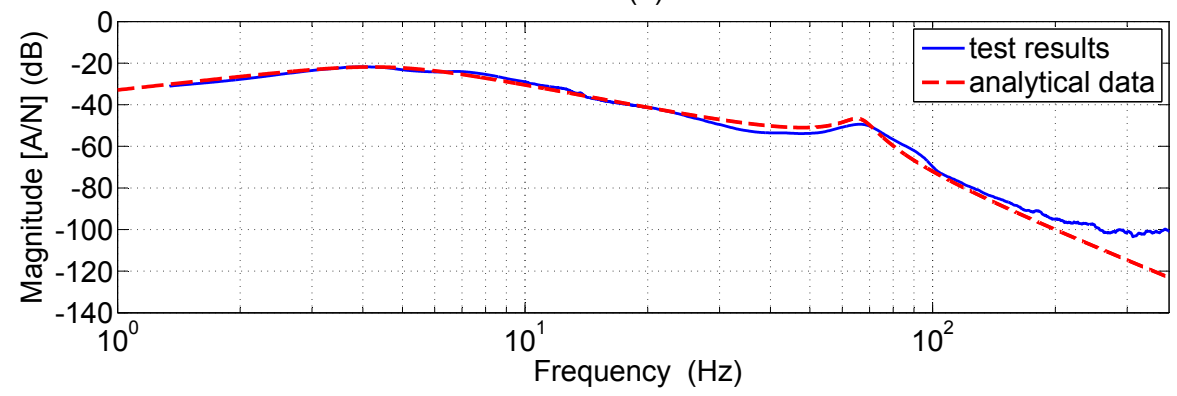

Figure 12: TF between the input force and the induced current in the electromagnets. The system considered is with EMSDs. (a) top electromagnet; (b) bottom electromagnet 
plates forming the dummy mass was changed from being in steel to being in aluminium. The overall supported mass $m_{1}$ was about $5.3 \mathrm{~kg}$. Four force transducers were placed between the reaction wheel and the dummy mass (see figure 9) and the total vertical input force generated by the wheel was measured by summing the signals of the four transducers. The rotational speed of the wheel was set to $1500 \mathrm{rpm}$. As shown in figure 13a, the input force ranges from $0.1 \mathrm{mN}$ at low frequency to about $5 \mathrm{mN}$ at high frequency. From a theoretical point of view, the two input forces (for the case with and without EMSD) should perfectly overlap since the wheel speed was the same. However, the small discrepancy that can be observed between the two curves might be due to speed errors introduced by the controller and time of recording.

The force TFs associated with the vertical force which was measured via the MFS and related to the system with and without EMSD is reported in figure 13b. It is noted that the disturbance force generated by the reaction wheel is at least a couple of orders of magnitude smaller than the input force applied through the mini-shaker. For this reason, it can be observed that the two TFs in figure 13b are characterised by a maximum attenuation of only $50 \mathrm{~dB}$ before hitting the background noise. However, both curves show good agreement with the results reported in the previous section thus demonstrating that the negative-resistance circuit is capable of working with broadband signals without showing any non-linearity or undesired behaviour.

\subsection{Performance evaluation}

In terms of damping performance, the 2-collinear-DoF isolator presented in this paper shows good advantages when compared to other damping solutions. The force TF of the system under examination (at the temperature of $15^{\circ} \mathrm{C}$ ) is reported in figure 14 along with the TF of a 1-DoF system with EMSD [31] and the one of a passive isolator that uses viscoelastic materials [1]. In particular, the 2-collinear-DoF system is capable of 


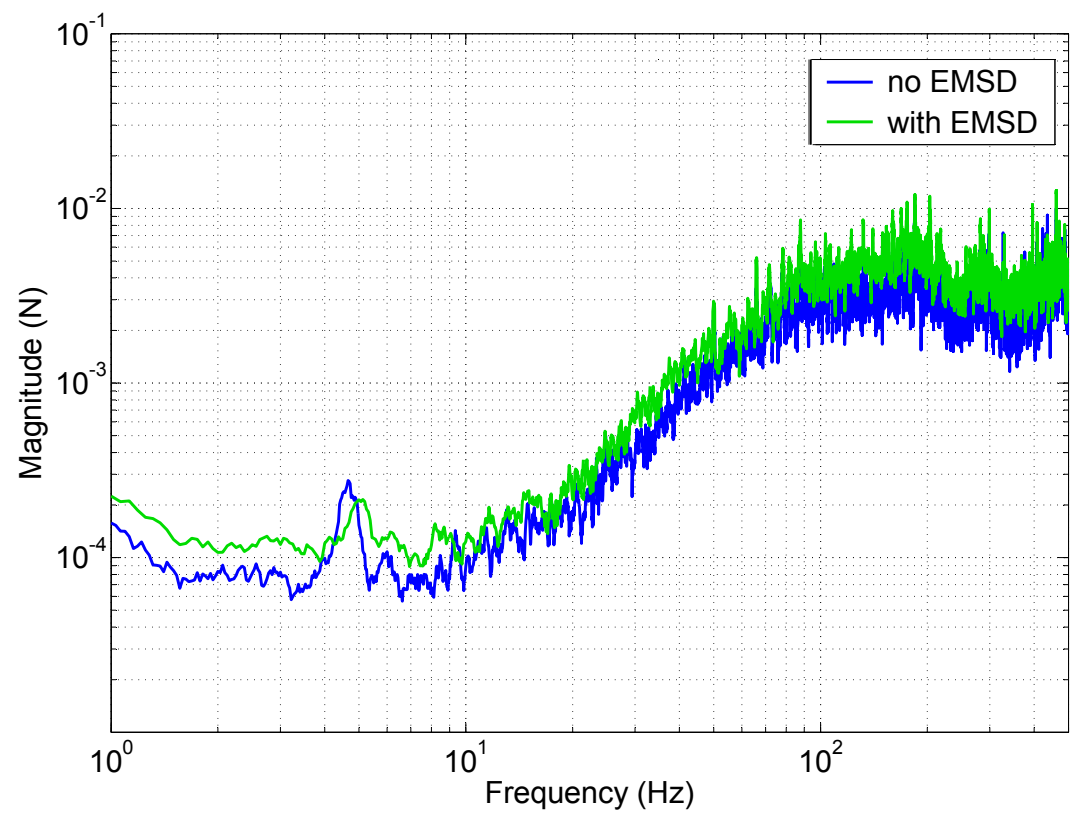

(a)

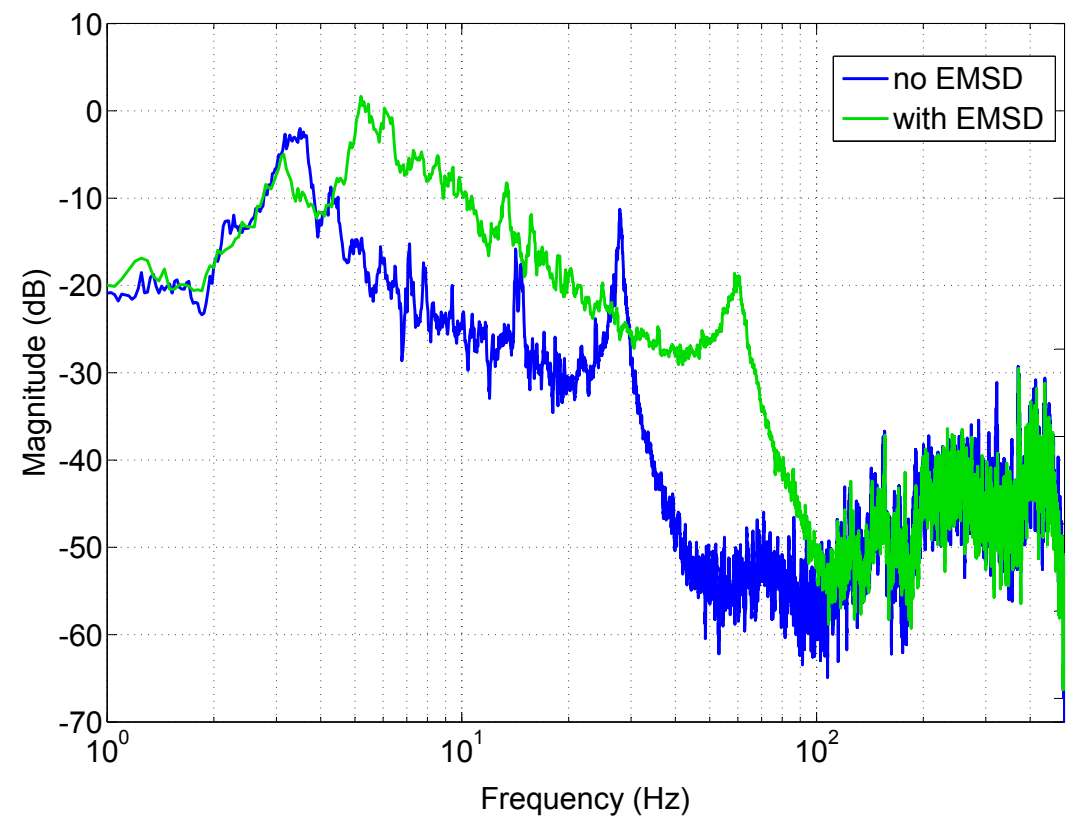

(b)

Figure 13: Experimental results for the test with the reaction wheel. (a) input force produced by the reaction wheel along the $z$ axis and measured by summing the signal of the four force transducers placed between the reaction wheel and the dummy mass; (b) TF between the input force and the vertical force measured with the MFS 
eliminating the resonance peak that is characteristic of a viscoelastic-material isolator and presents a final slope that is $40 \mathrm{~dB} / \mathrm{dec}$ greater than the one showed by the 1DoF system. Also in comparison with active dampers, the proposed strut shows several advantages. Active control methods are typically characterised by complex, cumbersome electronics that considerably affects the overall mass of the isolation system (e.g. the hexapod presented in [16] has an electronics mass of $9 \mathrm{~kg}$ which corresponds to 72 $\%$ of the overall hexapod mass) and require a significant amount of power to drive actuators and sensors (e.g. the single strut in [34] needs a minimum of $15 \mathrm{~W}$ to operate). Contrarily, the 2-collinear-DoF strut uses small circuit boards composed by few electric parts that require less than $0.5 \mathrm{~W}$ to produce the high damping performance reported in this paper.

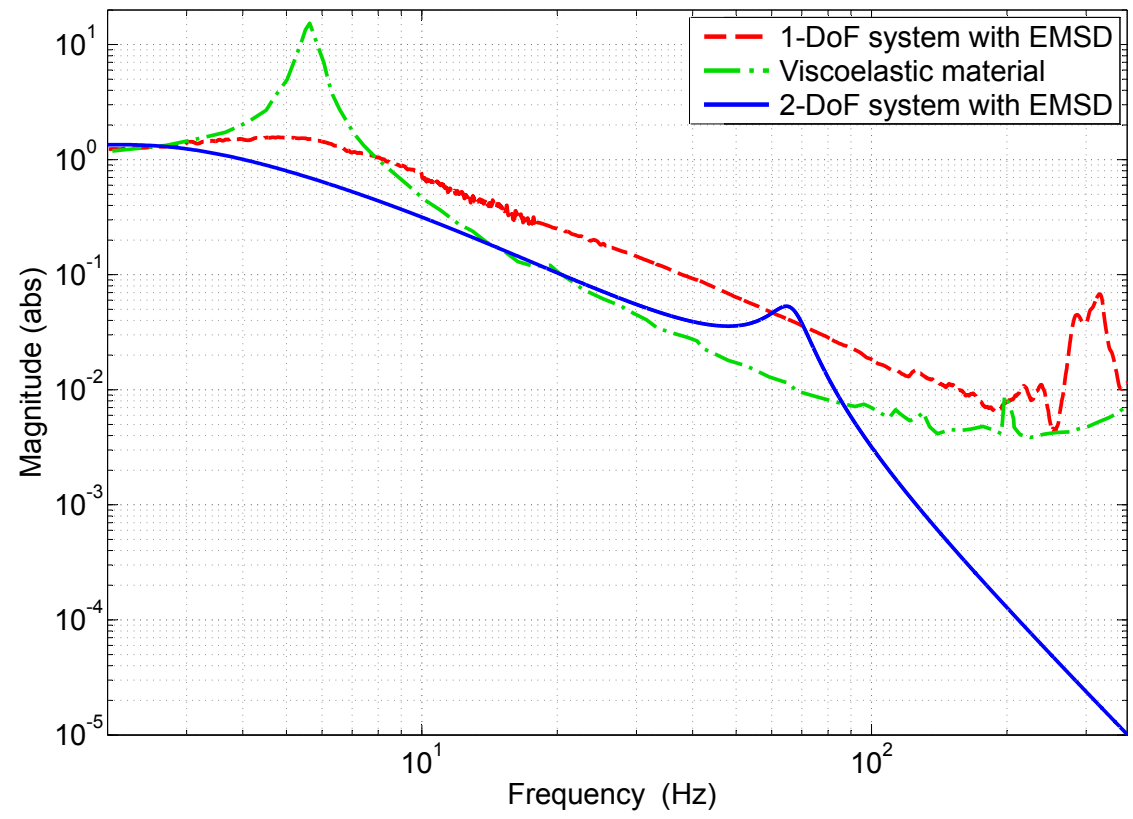

Figure 14: Comparison of the force TFs between three different damping systems: 1-DoF system with EMSD presented in [31], viscoelastic-material passive isolator presented in [1], and the 2-collinear-DoF system proposed in this paper. 


\section{Conclusions}

In the present work, a novel 2-collinear-DoF strut with embedded negative-resistance EMSDs has been studied both analytically and experimentally. The proposed isolator exploits the bipolarity of magnets to create a two-level damping that considerably enhances the isolation performance without the need to increase the secondary mass. The system state-space model has been presented, and a parametric trade-off has been carried out in order to meet the isolation requirements within the operating temperature range of $-20^{\circ} \mathrm{C}$ to $+50^{\circ} \mathrm{C}$. In particular, these goals included the limitation of the maximum disturbance amplification below $6 \mathrm{~dB}$, the corner frequency to be not greater than $10 \mathrm{~Hz}$ and an attenuation of at least $40 \mathrm{~dB}$ at $100 \mathrm{~Hz}$. The accuracy of the analytical model and the performance associated with the chosen parametric set were corroborated through an experimental campaign conducted on an in-house-built test rig. A sine sweep produced by a mini-shaker and a broadband signal generated by a real noise source (10SP reaction wheel) were applied to the supported mass $m_{1}$. This paper has demonstrated that the proposed strut is capable of producing better microvibration isolation than other well-established damping solutions without requiring an active control algorithm. Moreover, the small system mass, the simplified electronics and the little required power represent fundamental advantages for a space mission that will make this 2-collinear-DoF strut a viable, competitive alternative to other cumbersome actively-controlled struts.

\section{Acknowledgements}

The authors would like to thank Dr. Marcello Remedia for his valuable and constructive suggestions during the different phases of this research work 


\section{References}

[1] A.J. Bronowicki, R. MacDonald, Y Gursel, R. Goullioud, T. Neville, and D. Platus. Dual stage passive vibration isolation for optical interferometer missionsn. Proceedings SPIE Interferometry in space, 4852:753-763, Feb. 2003.

[2] G. Richardson, G. Smet, and G. Aglietti. Managing micro-vibration on the sstl300-s1 a $400 \mathrm{~kg} 1 \mathrm{~m}$ resolution earth imaging spacecraft. Proceeding of the 13th European Conference on Spacecraft Structures, Materials and Environmental Testing (SSMET), April 2014.

[3] G. Smet, G. Richardson, S. McLaren, and A Haslehurst. Managing reaction wheel microvibration on a high resolution eo small spacecraft. Proceeding of 15th European Space Mechanisms and Tribology Symposium, September 2013.

[4] P. Davis, D. Carter, and T.T. Hyde. Second generation hybrid d-strut. Proceeding of SPIE Smart Structures and Material conference, 1995.

[5] G.W. Wilson and P.J. Wolke. Performance prediction of d-strut isolation systems. Proceeding of SPIE Smart Structures and Material conference, 3045, 1997.

[6] C. Liu, X. Jing, S. Daley, and F. Li. Recent advances in micro-vibration isolation. Mechanical Systems and Signal Processing, 5657(0):55 - 80, 2015.

[7] G.S. Aglietti, S.B. Gabriel, R.S. Langley, and E. Rogers. A modelling technique for active control design studies with application to spacecraft microvibrations. Journal of The Acoustical Society of America, 102:2158-2166, 1997.

[8] G.S. Aglietti, R.S. Langley, E. Rogers, and S.B. Gabriel. An efficient model of an equipment loaded panel for active control design studies. Journal of The Acoustical Society of America, 108:1663-1673, 2000.

[9] G.S. Hauge and M.E. Campbell. Sensors and control of a space-based six-axis vibration isolation system. Journal of Sound and Vibration, 269(35):913 - 931, 2004.

[10] A. Preumont, M. Horodinca, I. Romanescu, B. de Marneffe, M. Avraam, A. Deraemaeker, F. Bossens, and A. Abu Hanieh. A six-axis single-stage active vibration isolator based on stewart platform. Journal of Sound and Vibration, 300(35):644 - 661, 2007.

[11] J. Spanos, Z. Rahman, and G. Blackwood. A soft 6-axis active vibration isolator. In American Control Conference, Proceedings of the 1995, volume 1, pages 412-416 vol.1, Jun 1995.

[12] D.-O. Lee, G. Park, and J.-H. Han. Hybrid isolation of micro vibrations induced by reaction wheels. Journal of Sound and Vibration, 363:1 - 17, 2016. 
[13] D.-O. Lee, G. Park, and J.-H. Han. Experimental study on on-orbit and launch environment vibration isolation performance of a vibration isolator using bellows and viscous fluid. Aerospace Science and Technology, 45:1 - 9, 2015.

[14] R.J. Cobb, J.M. Sullivan, A. Das, L.P. Davis, T.T. Hyde, T. Davis, Z.H. Rahman, and J.T. Spanos. Vibration isolation and suppression system for precision payloads in space. Smart Materials and Structures, 8(6):798, 1999.

[15] M. B. McMickell, T. Kreider, E. Hansen, T. Davis, and M. Gonzalez. Optical payload isolation using the miniature vibration isolation system (mvis-ii), 2007.

[16] J.H. Jacobs, J.A. Ross, S. Hadden, M. Gonzalez, Z. Rogers, and B.K. Henderson. Miniature vibration isolation system for space applications: Phase ii, 2004.

[17] R. Erwin, V. Babuska, L. Sullivan, and R. Fuentes. On-orbit active vibration isolation: the satellite ultraquiet isolation technologies experiment (suite). AIAA Space 2003 Conference and Exposition, September 2003.

[18] H-U Oh, J. Onoda, and K. Minesugi. Characteristics of a liquid-crystal type er-fluid variable damper for semiactive vibration suppression. Journal of vibration and acustics, 122(4), 2000.

[19] H-U Oh, J. Onoda, and K. Minesugi. Semiactive isolator with liquid-crystal type er fluid for momentum-wheel vibration isolation. Journal of vibration and acustics, 126(2), 2004.

[20] W. Zhao, B. Li, P. Liu, and K. Liu. semi-active control for a multi-dimensional vibration isolator with parallel mechasim. Journal of vibration and control, pages 1-10, 2012.

[21] J. Zheng, Z. Li, J. Koo, and J. Wang. Magnetic circuit design and multiphysics analysis of a novel mr damper for applications under high velocity. Advances in mechanical engineering, 2014.

[22] M. Unsal. semi-active vibration control of a parallel platform mechanism using magnetorheological damping. University of Florida, $\mathrm{PhD}$ thesis, 2006.

[23] S.M. Yang and C.A. Jeng. Structural vibration suppression by concurrent piezoelectric sensor and actuator. Smart Materials and Structures, 5(6):806, 1996.

[24] A.J. Fleming and S.O.R. Moheimani. Control oriented synthesis of electromagnetic shunt impedances for vibration isolation. IEEE transactions on Control Systems Technology, 13(1), 2005.

[25] J. J. Dosch, Daniel J. Inman, and E. Garcia. A self-sensing piezoelectric actuator for collocated control,. Journal of intelligent materials and structures, 3(1):166 - 185, 1992.

[26] A.J. Fleming, S.O.R. Moheimani, and S. Behrens. Synthesis and implementation of sensor-less 
active shunt controllers for electromagnetically actuated systems. Control Systems Technology, IEEE Transactions on, 13(2):246-261, March 2005.

[27] S Behrens, A.J. Fleming, and S.O.R. Moheimani. Control orientated synthesis of electromagnetic shunt impedances for vibration isolation. IEEE/ASME Transactions on Mechatronics, 9(1), 2004.

[28] X. Zhang, H. Niu, and B. Yan. A novel multimode negative inductance negative resistance shunted electromagnetic damping and its application on a cantilever plate. Journal of Sound and Vibration, 331(10):2257 - 2271, 2012.

[29] B. Yan, X. Zhang, Y. Luo, Z. Zhang, S. Xie, and Y. Zhang. Negative impedance shunted electromagnetic absorber for broadband absorbing: experimental investigation. Smart Materials and Structures, 23(12):125044, 2014.

[30] B. Yan, X. Zhang, and H. Niu. Design and test of a novel isolator with negative resistance electromagnetic shunt damping. Smart Materials and Structures, 21(3):035003, 2012.

[31] A. Stabile, G.S. Aglietti, G. Richardson, and G. Smet. Design and verification of a negative resistance electromagnetic shunt damper for spacecraft micro-vibration. Journal of sound and vibration, IN PRINT.

[32] A. Stabile, G.S. Aglietti, and G. Richardson. Electromagnetic damper design using a multiphysics approach. Proceeding of SPIE conference: Active and Passive Smart Structures and Integrated Systems, 9431, 2015.

[33] P. Horowitz and W. Hill. the art of electronics. Cambridge University Press, New York, 1996.

[34] E.H. Anderson, J.P. Fumo, and R.S. Erwin. Satellite ultraquiet isolation technology experiment (suite). In Aerospace Conference Proceedings, 2000 IEEE, volume 4, pages 299-313 vol.4, 2000. 